\title{
La escuela y los alumnos con déficit auditivo
}

\section{Marian Valmaseda}

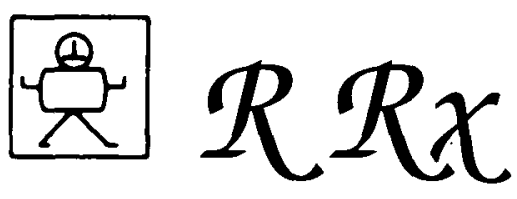

Los educadores que tienen a su cargo niños con deficiencias auditivas se enfrentan al problema de cómo bacerles acceder a la escolarización. En este artículo se proponen la evaluación inicial como berramienta imprescindible para adecuar la enseñanza a las necesidades específicas de cada alumno.

\section{CONSIDERACIONES PREVIAS}

Al abordar el tema de la educación de los alumnos con deficiencia auditiva nos enfrentamos a uno de los retos educativos más interesantes. Es éste un terreno en continuo desarrollo y, a la vez, en constante debate con enfoques muy diferentes y, en ocasiones, enfrentados.

¿Qué papel debe cumplir el lenguaje de signos en la educación de los alumnos sordos?, ¿qué ventajas e inconvenientes presenta la educación en un contexto educativo especial o de integración?, ¿existe una cultura y una comunidad sorda?, ¿qué características deben tener las interacciones comunicativas de los familiares del niño sordo para resultar más eficaces?, ¿es conveniente incluir adultos sordos en la educación de los niños y niñas con deficiencia auditiva?. Estas son algunas de las preguntas que están planteadas en relación a la educación de los alumnos sordos. No es nuestro objetivo abordar estas cuestiones en el presente escrito, sin embargo, consideramos importante que el lector tenga presente que el hecho de tratar el tema de la educación de los alumnos sordos tiene unas complejas implicaciones de tipo comunicativo-lingüístico y de tipo sociolingüístico que es preciso tomar en consideración.

Los diferentes enfoques educativos que podemos encontrar vienen determinados por la distintas respuestas que se dan a preguntas como las anteriormente planteadas, y por el hecho de que, cuando hablamos de alumnos con deficiencia auditiva, no hacemos referencia a un grupo homogéneo sino, por el contrario, a un grupo humano que presenta una gran diversidad, lo que hace posible, y necesario, un abordaje educativo desde diferentes enfoques.

Las diferencias individuales existentes entre los alumnos sordos son muy grandes y no sólo por la variedad en cuanto al tipo y grado de pérdida auditiva que presentan sino también, y sobre todo, porque una pérdida auditiva de cualquier grado interactúa de forma bastante impredecible con todos aquellos factores que permiten 
diferenciar unas personas de otras, factores tales como la personalidad, la motivación, sus habilidades e inhabilidades concretas así como las condiciones sociales, actitudinales y económicas de su entorno social y familiar.

\section{VALORACION DE LAS NECESIDADES EDUCATIVAS DE LOS ALUM- NOS SORDOS Y CRITERIOS PARA SU ESCOLARIZACION}

Por ello, a la hora de tomar decisiones respecto al contexto educativo más adecuado para un alumno en concreto, a las características y recursos que el mismo debe contar para ofrecer una adecuada respuesta educativa, será preciso realizar una valoración inicial de las características del propio alumno así como del contexto sociofamiliar y educativo en el que éste se desarrolla.

En lo que respecta a la información relativa al propio alumno es importante obtener datos referidos a: 1) su desarrollo biológico, intelectual, motor, lingüístico, emocional y de inserción social; 2) su nivel de competencia curricular y, 3) su estilo de aprendizaje y motivación para aprender.

Respecto a la evaluación de los diferentes aspectos del desarrollo, en el caso de los alumnos sordos, resulta de particular importancia conocer las condiciones auditivas, muy especialmente en lo relativo a las posibilidades de aprovechamiento de la audición residual. En estrecha relación con estos datos, será necesario obtener información relativa al desarrollo del área comunicativa-lingüística. Es preciso conocer con qué interlocutores (iguales y adultos) establece el alumno sordo intercambios comunicativos, cúal es la modalidad comunicativa más habitual en los mismos (oral, gestual, signada), y qué nivel de complejidad lingüística tienen esos intercambios, tanto en lo referente a la producción como a la comprensión.

La evaluación del nivel de competencia curricular implica determinar lo que el alumno es capaz de hacer en relación a los objetivos y contenidos de las diferentes áreas del currículo ordinario. Por su parte, la evaluación del estilo de aprendizaje y motivación para aprender implica tratar de conocer no sólo lo que es capaz de hacer el alumno sino también cómo aprende y qué le mueve a ello (Blanco et al. 1992). Obviamente, en el caso de los alumnos aún no escolarizados la evaluación de la competencia curricular así como del estilo de aprendizaje y motivación para aprender resultarán un tanto descontextualizada por lo que serán consideradas como una primera aproximación que habrá de ser completada en el centro escolar una vez ubicado el alumno.

Junto a los datos relativos a características del propio alumno es fundamental obtener información de su entorno socio-familiar, en particular, el estatus sordo/oyente de otros miembros de la familia, actitudes hacia la sordera de su hijo, expectativas de futuro y características del tipo de comunicación empleado en el hogar.

En lo que respecta al entorno escolar conviene conocer las experiencias educativas previas (en caso de haber existido) y valorar la oferta educativa existente en la zona, las características de los posibles centros, muy especialmente en lo que respecta a su proyecto educativo y a los recursos personales y materiales con que cuenta o puede contar para hacer frente a las particulares necesidades educativas de los alumnos sordos.

La combinación de la información obtenida tanto en lo que se refiere al propio alumno/a, como a su entorno conducirá a tomar la decisión de la opción educativa más adecuada en función de las características concretas de cada caso. A nuestro modo de ver, en esta decisión, es importante diferenciar entre: a) el tipo de respuesta educativa que es preciso ofrecer al alumno para hacer frente a sus necesidades educa- 
tivas especiales y favorecer su aprendizaje y, b) el contexto educativo en el que esta respuesta se ofrecerá.

Los datos obtenidos permitirán llevar a cabo una valoración inicial de las necesidades educativas que, en particular, presenta cada alumno sordo y a las cuales deberá ajustarse la respuesta educativa. A pesar de las diferencias que encontraremos entre las alumnas y alumnos sordos, es importante tener presentes algunas necesidades que todos ellos plantean al sistema educativo, necesidades que son, asímismo, comunes a cualquier otro alumno, pero que en nuestro caso toman especial relevancia:

1. Necesidad de un sistema de comunicación y pensamiento. Es decir, necesidad de apropiarse de forma temprana de un código que les permita establecer interacciones útiles y gratificantes con su entorno a la vez que pueda servirles como herramienta de representación, de simbolización del mundo que le rodea y, por tanto, de aprendizaje.

2. Necesidad de mayor información de lo que sucede en su entorno. Dadas sus limitaciones auditivas los alumnos sordos tendrán un menor acceso a la información auditiva que constántemente nos rodea. Para paliar esta situación, el entorno debe proporcionar, de forma intencional, información de todo tipo incluyendo la referida a normas, valores y actitudes.

3. Necesidad de un desarrollo emocional lo más equilibrado posible, desarrollando una identidad y autoconcepto positivo.

La consideración de estas necesidades comunes junto a las particulares de cada alumno deben guiar la toma de decisiones en lo que respecta a la escolarización más adecuada y, sobre todo, en cuanto al tipo y calidad de los ajustes que será preciso realizar en la propuesta educativa ofrecida.

\section{OBJETIVOS Y CONTENIDOS PRIORITARIOS DURANTE LA ESCOLARIZACION OBLIGATORIA}

Generalmente, cuando los niños y niñas con pérdidas auditivas llegan a la escuela lo hacen con menos experiencias y con un bagage de información más limitado que en el caso de los alumnos oyentes. Por otra parte, presentan, en mayor o menor grado un deficiente manejo de la lengua oral empleada en su entorno. Esta diferencia en cuanto a los "previos" con los que el niño con déficit auditivo llega a la escuela hace que sea necesario llevar a cabo una serie de modificaciones en la respuesta educativa. Estas modificaciones tendrán lugar, prioritariamente, en los instrumentos de acceso al currículo aunque también será necesario llevar a cabo modificaciones en el propio currículo.

1. La primera decisión a tomar a la hora de dar respuesta educativa a las alumnas y alumnos sordos irá dirigida a establecer con los mismos, cauces comunicativos que posibiliten su acceso a los objetivos y contenidos curriculares. En el contexto escolar el lenguaje oral y el lenguaje escrito son considerados, a la vez, como instrumentos de acceso al currículo y como aprendizajes a desarrollar y enriquecer a partir de su uso en contextos diversos y en relación a diferentes materias. No es, pues, de extrañar que en la respuesta educativa para los alumnos sordos, el aprendizaje/enseñanza de la lengua oral se convierta en un objetivo prioritario.

Ahora bien, en el caso de los alumnos con graves pérdidas auditivas, este aprendizaje, a pesar de los esfuerzos de profesores y alumnos, sólo se produce de manera limitada e imperfecta por lo que el alumno contará con un instrumento de acceso al currículo pobre y ello, a su vez, limitará sus posibilidades de acceso cultural. Por ello, en la mayoría de los alumnos con graves pérdidas auditivas, será preciso 
emplear sistemas complementarios o alternativos a la lengua oral (Bimodal, Palabra Complementada, Lengua de signos etc..) sin olvidar la especial relevancia que el aprendizaje de la lecto-escritura tiene a lo largo del proceso educativo.

2. En el caso de los alumnos sordos resulta de particular importancia la explicitación de contenidos referidos a valores, normas y actitudes. Recordemos que los alumnos sordos tienen menores oportunidades de conocer las normas que rigen su entorno, así como de poner en discusión sus opiniones y actitudes.

3. Introducción de contenidos acerca de la sordera. Un centrọ que eduque alumnos sordos, sea en un contexto de integración o de educación especial, debe introducir en su propuesta curricular contenidos acerca de la sordera. Estos contenidos pueden ser diversos y abordar, por ejemplo, el funcionamiento del oído, las carácterísticas físicas y de transmisión del sonido, el modo en que las personas sordas se comunican entre sí, las diferencias entre las lenguas de signos y las lenguas orales, las implicaciones que puede tener la dificultad o ausencia de audición en la vida cotidiana, cúales son las manifestaciones culturales de las personas sordas, etc.... Estos contenidos pueden ser introducidos de forma transversal en las distintas áreas del currículo, ofreciendo a los alumnos la posibilidad de conocer y discutir aspectos importantes que interesan no sólo a los alumnos sordos sino también a sus compañeros oyentes. A menudo, unos y otros desconocen las implicaciones de una pérdida auditiva así como las características vitales y sociales de las personas sordas adultas. Para todos ellos resultará importante tener posibilidades de contacto e interacción con iguales y adultos sordos y oyentes.

4. En muchos casos, aunque alumnos y profesores cuenten con un instrumento de comunicación a través del cual llevar a cabo los procesos de enseñanza/aprendizaje, se hace necesario modificar algunos contenidos de áreas curriculares. Obviamente, ello dependerá de la competencia curricular de cada alumno en particular; no obstante algunas áreas plantean "a priori" mayores dificultades. Este es el caso del área de Lengua y Literatura, Lenguas Extranjeras y los contenidos de Música en el Area de Educación Artística.

$\mathrm{El}$ área de Lengua y Literatura sufrirá importantes modificaciones ya que debe incorporar la enseñanza intencional de objetivos y contenidos que se consideran previos en los alumnos oyentes. Nos referimos de forma muy particular a la enseñanza/aprendizaje de la lengua oral aunque también la enseñanza de la lectoescritura puede ser objeto de importantes modificaciones.

En lo que respecta al aprendizaje de la Lengua Extranjera, las decisiones respecto a las posibles adaptaciones deberán tomarse en función de las habilidades linguiísticas ya desarrolladas por el propio alumno. En general, es importante priorizar aquellos objetivos y contenidos referidos a la comprensión y producción de la lengua en su modalidad escrita. En algunos casos será necesario realizar adaptaciones muy significativas manteniendo únicamente algunos objetivos relativos al aprendizaje de un vocabulario básico o de expresiones de alto valor funcional.

En cuanto a los objetivos y contendios de música del área de Educación Artística precisarán adaptaciones en función de las posibilidades auditivas de cada alumno. En principio, hemos de partir de la idea básica de que el alumno sordo, en la medida de sus posibilidades, puede y debe participar en las actividades musicales. Aún en el caso de alumnos con graves pérdidas auditivas, es posible trabajar aspectos básicos del ritmo y de la percepción de sonidos a través de la estimulación vibro-tactil. Junto a ello, será importante conceder especial relevancia a los objetivos y contenidos del apartado de Lenguaje Corporal y de Juego Dramático asímismo incluidos en el área de Educación Artística. 


\section{ALGUNAS ESTRATEGIAS METODOLOGICAS Y ORGANIZATIVAS}

Los ajustes educativos deberán realizarse no sólo en los objetivos y contenidos de enseñanza/aprendizaje, sino también, y sobre todo, en el modo en que este proceso se lleva a cabo. A la hora de plantear estrategias de enseñanza con alumnos sordos es importante tener muy en cuenta cuál es el canal sensorial en el que estos alumnos se basan, de manera prioritaria, para acceder a la información. En este sentido, podemos identificar dos grupos diferenciados: los que pueden acceder a la información a través del canal auditivo y los que deben basarse principalmente en la visión. Estos diferentes modos de acceso a la información tienen importantes repercusiones en lo que respecta a la modalidad del lenguaje que estos alumnos pueden desarrollar de manera natural, a las características de sus intercambios comunicativos y, por tanto, a las estrategias y recursos materiales y personales necesarios para llevar adelante el proceso educativo.

Sin ánimo de ser exhaustivos, comentaremos algunas de las estrategias que pueden favorecer el proceso de enseñanza/ aprendizaje de los alumnos sordos en el aula.

a) En primer lugar, es importante tener presente que ciertas decisiones metodológicas y organizativas serán facilitadoras, no del aprendizaje de los alumnos ' sordos, sino de cualquier alumno. Poner en práctica una metodología y una organización que permịta la flexibilidad y la implicación activa de los alumnos beneficiará el aprendizaje de todos los alumnos.

b) Las condiciones para el aprendizaje deben ser modificadas de forma que los alumnos y alumnas con pérdidas auditivas puedan participar en las actividades de enseñanza/aprendizaje. En este sentido es importante optimizar las condiciones acústicas y de iluminación del aula, cuidar la ubicación del alumno y, asegurar un adecuado manejo de las ayudas técnicas empleadas, en particular la prótesis auditiva del alumno y, en su caso, los equipos de frecuencia modulada.

c) Siempre que sea posible conviene que la información le sea presentada al alumno o alumna sorda de manera visual por ejemplo a través de diagramas, esquemas, texto escrito, visualización de vídeos, diapositivas, transparencias etc...

d) Es conveniente explicitar a los alumnos el proceso de enseñanza; esto es, informarles acerca de lo que se va a trabajar y por qué, en qué consiste la actividad, qué se espera que haga el alumno y qué el profesor, cómo se evaluará lo aprendido etc... Si todo ello es importante en el caso de los alumnos oyentes, lo es aún más en el caso de los alumnos sordos debido a sus menores posibilidades de acceder a la información lo que, a menudo, se traduce en desorientación y desconcierto respecto a lo que sucede en su entorno. La información puede ofrecerse de forma oral, escrita, signada, a través de dibujos, con ejemplos etc, dependiendo de las necesidades de los alumnos del aula.

e) Las niñas y niños sordos aprenden mejor cuando se les permite implicarse activamente en el proceso de aprendizaje. Para ello es importante potenciar la experiencia directa promoviendo actividades de exploración, observación, demostraciones, representaciones drámaticas etc..

f) Es importante favorecer las interacciones con otros compañeros. Un buen número de nuestros aprendizajes son el resultado de la interacción con compañeros, con iguales, especialmente en el marco de la cooperación. Planificar actividades que pueden ser resueltas de forma cooperativa será de gran ayuda para los alumnos sordos. En los contextos de integración escolar es importante que los compañeros oyentes sean sensibles a las necesidades comunicativas de sus compañeros sordos y modifiquen sus estrategias comunicativas para facilitar, así, las interacciones. Junto a ello, desde un punto de vista organizativo, será importante organizar grupos diferentes en 
función de distintas actividades ofreciendo al alumno sordo la posibilidad de interactuar tanto con compañeros oyentes como con compañeros sordos.

g) Es importante que los alumnos sordos se enfrenten al aprendizaje sintiéndose aceptados y capaces de aprender. En este sentido, el papel que el profesor juega resulta central. Sus expectativas hacia los alumnos sordos, el tipo de atribuciones para con las consecuciones de los mismos, pueden mejorar o, por el contrario, empobrecer aún más la autoimagen que los alumnos sordos tienen de sí mismos. Como hemos señalado, muchos niños con pérdidas auditivas acuden a la escuela con pobres experiencias, limitado lenguaje y poca confianza en sí mismos. La escuela debe propiciar situaciones de éxito y refuerzo positivo para contrarestrar los sentimientos negativos de los alumnos. Para ello es importante identificar las capacidades de los alumnos y no sólo sus "debilidades", planificar actividades que los alumnos puedan llevar a cabo y evitar comparaciones de unos alumnos con otros.

h) En muchas ocasiones será necesario adaptar los materiales escritos que se vayan a emplear. Estas adaptaciones pueden ser de distintos tipos. Pueden, por ejemplo, incorporar mayor información visual a través de dibujos y gráficos o bien a través de signos que acompañan al texto escrito (CNREE, 1990). Sin embargo las adaptaciones más habituales son las que tienen lugar en el propio mensaje escrito, principalmente en el vocabulario y en ciertas estructuras morfo-sintácticas. Debemos tratar que los textos escritos sean apropiados a la edad e intereses de los alumnos sordos lo que no siempre resulta sencillo debido a la distancia que algunos alumnos muestran entre su edad cronológica y la edad que correspondería a su nivel lingüístico. En estos casos, los textos correspondientes al nivel lingúístico pueden resultar excesivamente infantiles tanto en lo que respecta a las características del estilo como a su contenido por lo que un excesivo empleo de los mismos puede reforzar, aún más, el auto-concepto negativo de los alumnos sordos a la vez que no potencia su aprendizaje. Por ello, siempre que sea posible, resultará más adecuado adaptar los materiales escritos intentando que éstos reflejen tanto las habilidades lingüísticas de los alumnos como sus intereses personales.

\section{CRITERIOS Y PROCEDIMIENTOS PARA LA EVALUACION}

Las decisiones acerca de los criterios de evaluación que van a tomarse en consideración así como la selección de procedimientos para llevarla a cabo deben estar presentes desde el inicio en la Programación de Aula y, en su caso en Documento que refleja las Adaptaciones Curriculares de un alumno concreto. Se trata, en definitiva, de evaluar aquello que se pretende que el alumno aprenda. La evaluación debe informarnos acerca de la consecución de los objetivos y contenidos programados, de cómo el alumno se enfrenta a la tarea (estilo de aprendizaje) así como de su motivación para aprender. En el caso de que el alumno no haya alcanzado los objetivos y contenidos propuestos será necesario analizar las posibles causas, principalmente en lo que respecta a las actividades planteadas para llevar a cabo tal aprendizaje, a las estrategias metodológicas etc... en definitiva se tratará de evaluar aquellos elementos del contexto que pueden ser susceptibles de modificación.

El principal riesgo que, a nuestro modo de ver, entraña la evaluación del aprendizaje de los alumnos sordos es el de no diferenciar claramente entre la evaluación de los objetivos y contenidos alcanzados y la evaluación del nivel lingüístico. Señalábamos al comienzo que el proceso de enseñanza/aprendizaje no puede tener lugar si no existe un sistema comunicativo compartido entre los diferentes agentes implicados en el mismo. Pues bien, nuevamente nos enfrentamos al mismo problema. En la medida en que tengamos dificultades para compartir con nuestros alum- 
nos sordos un sistema de comunicación eficaz, nos enfrentaremos, asímismo, con dificultades para poder llevar a cabo la evaluación de sus aprendizajes.

Siempre que la evaluación se realice a través de la lengua oral o escrita debemos ser muy cuidadosos en aislar el "fondo" de la "forma" ya que en caso contrario estaríamos, sin darnos cuenta, evaluando una y otra vez el nivel lingüístico de nuestro alumno sordo. Por ello es importante emplear estrategias evaluadoreas diversas tales como observaciones, pruebas escritas, representaciones gráficas, dramatizaciones, demostraciones etc...

\section{A MODO DE CONCLUSION}

Aunque las consideraciones realizadas se han situado en relación a la respuesta educativa que tiene lugar en el Aula, es importante tener en cuenta que la educación de los alumnos sordos no debe ser una tarea individual o independiente de unos profesores respecto a otros sino que, por el contrario, debe ser el resltado de unas propuestas comunes articuladas a través del Proyecto Educativo. Las necesidades educativas de los sordos deben modular los diferentes elementos del Proyecto Educativo del Centro de forma que aquellas sean tenidas en cuenta en cualquier decision educativa que se adopte.

Cada alumna o alumno sordo planteará diferentes necesidades y precisará de una respuesta específica. Sin embargo, estamos de acuerdo con Johnson et al. (1989) cuando plantean que la clave del éxito educativo con los alumnos sordos pasa por proporcionar a estos alumnos un sistema de acceso lingüístico a los contenidos escolares y por romper el ciclo de bajas expectativas con respecto a sus posibilidades de aprender.

\section{Referencias}

Blanco, R.; Sotorrio, B.; Rodriguez, V.; Pinto, T.; Diaz-Estébanez, E., y Martin, M. (1992). Alumnos con necesidades educativas especiales y adaptaciones curriculares. Madrid: Centro Nacional de Recursos para la Educación Especial. M.E.C.

CNREE (1990). La casita de chocolate. Serie Cuentos en Comunicación Bimodal. Madrid: Centro Nacional de Recursos para la Educación Esepcial. M.E.C.

JOHNSON, R. E.; LIDDELL, S. K., y ERTING, C. J. (1989). Unlocking the curiculum: Principles for achieving acces in deaf education. Washington D.C.: Gallaudet Rersearch Institute.

\section{Bibliografía básica. Sugerencia para los lectores}

No resulta fácil elaborar una bibliografía básica que aborde el tema de la educación de los alumnos sordos. La mayor parte de las publicaciones existentes se centran en el ámbito comunicativo y más concretamente en la intervención logopédica. Proponemos al lector un conjunto de referencias que, tomadas globalmente, pueden ayudar a establecer una visión general de cuestiones relativas al desarrollo cognitivo, comunicativo y socio-afectivo de estos alumnos junto a consideraciones educativas.

Alonso, P., y Valmaseda, M. (1993). Los sistemas de comunicación sin ayuda. En M. Sotillo Los sistemas de comunicación. Madrid: Ediciones Trotta.

GARRIDO, C.; VASQUEZ, A., y MARTINEZ, I. (1990). La integración de los niños sordos en el sistema escolar. El caso de Adela G. Infancia y Aprendizaje, 50, 43-62 
LuCAS, C. (1989). The saciolinguistics of the Deaf Community. San Diego: Academic Press.

MARCHESI, A. (1987). El desarrollo cognitivo y lingüístico de los niñossordos. Madrid: Alianza Psicología.

MARTINEZ, I. (1990). El desafío de la Integración. Barcelona: Ediciones Milán y Fundació Catalana per a la Sindrome de Down.

Neal Davies, S. (1991). The transition toward Bilingual education of deaf children in Sweden and Denmark: Perspectives on Language. Sign Language studies, 71, 169-195

SANDERS, D. (1988). Teaching deaf children: Texhniques and methods. Boston: College-Hill Publication.

STONE HARRIs, R. (1988). Let's learn about deafness. Washington D.C.:Gallaudet Press. (Traducción española (1992) Aprendiendo cosas sobre la sordera: actividades para el aula. Madrid: Centro Nacional de Recursos para la Educación especial. M.E.C.)

VVAA (1991). Las necesidades educativas especiales del niño con deficiencia auditiva. Serie Formación. Madrid: Centro Nacional de Recursos para.la Educación Especial. M.E.C.

\section{La escuela y los alumnos con déficit auditivo Marian Valmaseda CL\&E, 1994, 22, pp. 7-14}

Resumen: A lo largo de este escrito se ofrecen algunas consideraciones relativas a la respuesta educativa dirigida a aquellos alumnos y alumnas que presentan deficiencias auditivas. Se aboga por la valoración inicial que permita llevar a cabo una primera determinación de las necesidades educativas especiales de cada alumno y tomar decisiones respecto a la escolarización más adecuada. Una vez que el alumno esté escolarizado será preciso realizar modificaciones tanto en los medios que permiten el acceso al currículo como en el propio currículo. Comentaremos algunas de estas adaptaciones situándonos en la perspectiva del profesor en el Aula.

Datos sobre la autora: Marian Valmaseda es licenciada en Psicología y titulada en Psicología del Lenguaje. Actualmente coordina el Departamento de Deficiencia Auditiva del Centro Nacional de Recursos para la Educación Especial de Madrid.

Dirección: C.N.R.E.E. C/ General Oráa, 55, 28006 Madrid.

(C) PERMISOS PARA CITAR O REPRODUCIR EN OTRAS FUENTES: Se pueden citar libremente hasta 500 palabras. Para reproducir una porción de texto mayor, figuras o ilustraciones, se deberá pedir permiso por escrito a la revista, especificando el uso al que se destina el texto. En todos los casos, se deberá citar el copyright de $C L \& E$. En el caso de artículos o textos que hayan sido a su vez reproducidos en $C L \& E$ los interesados deberán dirigirse tanto a los detentadores del copyright original como a $C L \& E$, en el caso de que se quiera hacer uso de la traducción. FOTOCOPIAS: Para todo lo relacionado con el uso mediante fotocopia del material de esta revista, deberán dirigirse a: CEDRO, C/ José Marañón, 10, 3. ${ }^{\circ}$ Izda. Tel. 5941575 . Fax 4453567 